\title{
A Tandem Non-Aldol Aldol Mukaiyama Aldol Reaction
}

Michael E. Jung* and Alexandra van den Heuvel

Department of Chemistry and Biochemistry, University of California, Los Angeles, CA 90095-

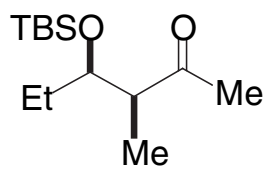

(3S,4R)-4-(1,1-dimethylethyldimethylsilyloxy)-3-methyl-hexan-2-one (6)

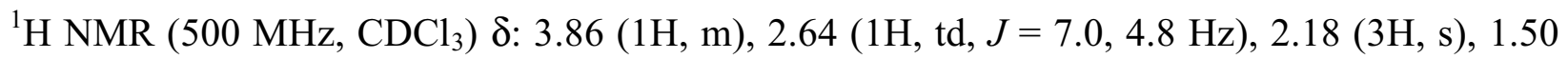
$(1 \mathrm{H}, \mathrm{m}), 1.39(1 \mathrm{H}, \mathrm{m}), 1.06(3 \mathrm{H}, \mathrm{d}, J=7.0 \mathrm{~Hz}), 0.88(9 \mathrm{H}, \mathrm{s}), 0.87(3 \mathrm{H}, \mathrm{t}, J=7.4 \mathrm{~Hz}), 0.06(3 \mathrm{H}$, s), $0.04(3 \mathrm{H}, \mathrm{s})$.

${ }^{13} \mathrm{C}$ NMR $\left(126 \mathrm{MHz}, \mathrm{CDCl}_{3}\right) \delta: 211.7,74.7,51.5,30.0,27.4,25.8(3 \mathrm{C}), 18.1,11.5,9.8,-4.3$, 4.6.

IR(neat):2957, 2859, 1713, 1464, 1258, $1045 \mathrm{~cm}^{-1}$.

HRMS (EI, $m / z): 187.115784$, calc for $\mathrm{C}_{9} \mathrm{H}_{19} \mathrm{O}_{2} \mathrm{Si}(\mathrm{M}-t \mathrm{Bu})^{+} 187.115433$.

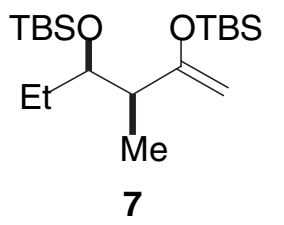

(3S,4R)-2,4-Bis-(1,1-dimethylethyldimethylsilyloxy)-3-methyl-hex-1-ene (7)

${ }^{1} \mathrm{H}$ NMR $\left(400 \mathrm{MHz}, \mathrm{CDCl}_{3}\right) \delta: 4.02(1 \mathrm{H}, \mathrm{d}, J=0.8 \mathrm{~Hz}), 3.96(1 \mathrm{H}, \mathrm{d}, J=0.8 \mathrm{~Hz}), 3.73(1 \mathrm{H}, \mathrm{m})$, $2.18(1 \mathrm{H}, \mathrm{td}, J=6.9,6.9 \mathrm{~Hz}), 1.54(2 \mathrm{H}, \mathrm{m}), 1.02(3 \mathrm{H}, \mathrm{d}, J=6.8 \mathrm{~Hz}), 0.92(9 \mathrm{H}, \mathrm{s}), 0.89(9 \mathrm{H}, \mathrm{s})$, $0.86(3 \mathrm{H}, \mathrm{t}, J=7.4 \mathrm{~Hz}), 0.17(3 \mathrm{H}, \mathrm{s}), 0.16(3 \mathrm{H}, \mathrm{s}), 0.03(3 \mathrm{H}, \mathrm{s}), 0.02(3 \mathrm{H}, \mathrm{s})$. 
${ }^{13} \mathrm{C}$ NMR $\left(126 \mathrm{MHz}, \mathrm{CDCl}_{3}\right) \delta: 161.5,89.1,73.9,44.6,27.7,26.0,25.7,18.2,18.1,14.6,8.5,-$ $4.3,-4.6,-4.8,-4.9$.

IR (neat): 2930, 1620, 1474, 1256.

\section{General procedure for the non-aldol aldol Mukaiyama reaction and non-enolizable} aldehydes

In an oven dried $10 \mathrm{~mL}$ flask was placed the epoxy alcohol 8 (1eq.), dichloromethane $(0.1 \mathrm{M})$ and powdered $4 \AA$ molecular sieves. The solution was cooled to $-40{ }^{\circ} \mathrm{C}$ and $i \operatorname{Pr}_{2} \mathrm{NEt}$ (1.35 eq.) followed by $t$-butyldimethylsilyl triflate $(1.3$ eq.) were added. The reaction mixture was stirred at $-40{ }^{\circ} \mathrm{C}$ for $2 \mathrm{~h}$ and the temperature was allowed to rise to $-20{ }^{\circ} \mathrm{C}$. The flask was then left in the freezer overnight. The reaction mixture was cooled to $-78{ }^{\circ} \mathrm{C}$ and the aldehyde (2 eq.) and $t$ butyldimethylsilyl triflate $(5 \mathrm{~mol} \%)$ were added. The reaction was allowed to warm to $21{ }^{\circ} \mathrm{C}$ over $5 \mathrm{~h}$. The reaction was quenched with $\mathrm{pH} 7$ buffer, extracted with dichloromethane, washed with $\mathrm{pH} 7$ buffer and brine. The organic phase was dried over $\mathrm{MgSO}_{4}$, filtered and concentrated under vacuo to give a yellowish liquid. The crude ketone was purified by flash column chromatography ( $\mathrm{SiO}_{2}$, pentane/ether: $99 / 1$ to $98 / 2$ ).

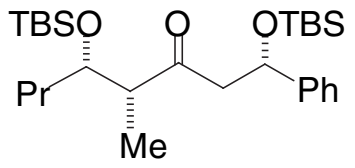

$(1 S, 4 R, 5 S)$-1,5-Bis-(1,1-dimethylethyldimethylsilyloxy)-4-methyl-1-phenyl-octan-3-one (10)

Started with the epoxide $8(500 \mathrm{mg}, 1.94 \mathrm{mmol})$ and obtained the protected 1,5-diol in $63 \%$ yield with a 4/1 syn/anti diastereomeric ratio.

${ }^{1} \mathrm{H}$ NMR (500 MHz, $\left.\mathrm{CDCl}_{3}\right)$ \&: $7.29(4 \mathrm{H}, \mathrm{m}), 7.23(1 \mathrm{H}, \mathrm{m}), 5.18(1 \mathrm{H}, \mathrm{dd}, J=7.8,4.7 \mathrm{~Hz}), 3.82$ $(1 \mathrm{H}, \mathrm{m}), 3.11(1 \mathrm{H}, \mathrm{dd}, J=16.7,7.9 \mathrm{~Hz}), 2.64(1 \mathrm{H}, \mathrm{dd}, J=16.7,4.7 \mathrm{~Hz}), 2.48(1 \mathrm{H}, \mathrm{m}), 1.30(4 \mathrm{H}$, 
m), $0.95(3 \mathrm{H}, \mathrm{d}, J=7.0 \mathrm{~Hz}), 0.87(9 \mathrm{H}, \mathrm{s}), 0.85(3 \mathrm{H}, \mathrm{t}, J=7.2 \mathrm{~Hz}), 0.83(9 \mathrm{H}, \mathrm{s}), 0.04(3 \mathrm{H}, \mathrm{s})$, $0.03(3 \mathrm{H}, \mathrm{s}), 0.02(3 \mathrm{H}, \mathrm{s}),-0.18(3 \mathrm{H}, \mathrm{s})$.

${ }^{13} \mathrm{C}$ NMR (126 MHz, $\left.\mathrm{CDCl}_{3}\right) \delta: 210.7,145.0,128.2(2 \mathrm{C}), 127.2,126.0(2 \mathrm{C}), 73.5,70.9,53.8$, $52.2,36.8,25.9(3 \mathrm{C}), 25.8(3 \mathrm{C}), 18.6,18.1,14.2,12.0,-4.37,-4.42,-4.8,-5.1 /$ (one upfield carbon not observed).

IR (neat): 3032, 2957, 2859, 1715, 1464, $1258 \mathrm{~cm}^{-1}$.

\section{General procedure for the Mukaiyama aldol using dimethylphenylsilyllithium}

Preparation of the dimethylphenylsilyllithium: In an oven dried $25 \mathrm{~mL}$ flask was placed litium shots $(81.5 \mathrm{mg}, 11.75 \mathrm{mmol})$ and dry hexane $(4 \mathrm{~mL})$. The suspension was stirred under argon for $10 \mathrm{~min}$ and then sonicated for $5 \mathrm{~min}$ and the hexane was removed via syringe. To the activated lithium was added tetrahydrofuran $(3.36 \mathrm{~mL})$. The suspension was cooled to $0{ }^{\circ} \mathrm{C}$, dimethylphenylchlorosilane $(839 \mu \mathrm{L}, 5 \mathrm{mmol})$ was added and the reaction was stirred at $0{ }^{\circ} \mathrm{C}$ for $6 \mathrm{~h}$. The deep red solution was kept in the freezer overnight. The solution was titrated with $\mathrm{HCl}$ $(0.102 \mathrm{M})$ and was found to be $0.86 \mathrm{M}$.

In an oven dried $10 \mathrm{~mL}$ flask was placed the silyl enol ether 9 (100 mg, $0.27 \mathrm{mmol}), \mathrm{C}_{6} \mathrm{D}_{6}(150$ $\mu \mathrm{L})$ and tetrahydrofuran $(1 \mathrm{~mL})$. The dimethylphenylsilyllithium (2 eq.) was added and the deep red solution was stirred for $2 \mathrm{~min}$ and then the reaction was allowed to sit for $4 \mathrm{~h}$. The reaction mixture turned yellow and was cooled to $-78{ }^{\circ} \mathrm{C}$. The aldehyde (2 eq.) was added and the reaction was stirred for $30 \mathrm{~min}$. The reaction was quenched by pouring it into sat. $\mathrm{NH}_{4} \mathrm{Cl}(1.5$ $\mathrm{mL}$ ). The mixture was extracted with diethyl ether, the combined organic phase was washed with brine, dried over $\mathrm{MgSO}_{4}$ and concentrated in vacuo to give a yellow liquid. The crude ketol was purified by flash column chromatrography $\left(\mathrm{SiO}_{2}\right.$, pentane/ether: $9 / 1$ to $\left.85 / 15\right)$. 


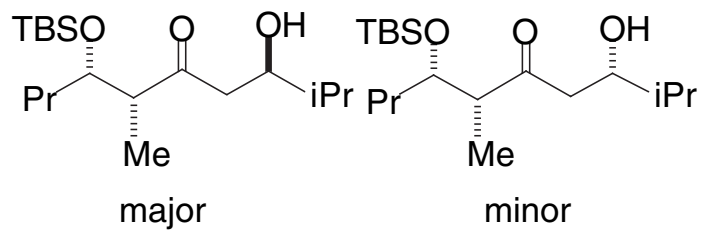

7-(1,1-dimethylethyldimethylsilyloxy)-3-hydroxy-2,6-dimethyl-decan-5-one (11a)

Major isomer:

${ }^{1} \mathrm{H}$ NMR $\left(500 \mathrm{MHz}, \mathrm{CDCl}_{3}\right) \delta: 3.88(1 \mathrm{H}, \mathrm{m}), 3.77(1 \mathrm{H}, \mathrm{m}), 3.17(1 \mathrm{H}, \mathrm{d}, J=3.1 \mathrm{~Hz}), 2.67(1 \mathrm{H}$, qd, $J=7.0,4.4 \mathrm{~Hz}), 2.63(2 \mathrm{H}, \mathrm{m}), 1.66(1 \mathrm{H}, \mathrm{m}), 1.32(4 \mathrm{H}, \mathrm{m}), 1.05(3 \mathrm{H}, \mathrm{d}, J=7.0 \mathrm{~Hz}), 0.93$ $(3 \mathrm{H}, \mathrm{d}, J=6.8 \mathrm{~Hz}), 0.90(3 \mathrm{H}, \mathrm{d}, J=6.8 \mathrm{~Hz}), 0.88(9 \mathrm{H}, \mathrm{s}), 0.87(3 \mathrm{H}, \mathrm{m}), 0.06(3 \mathrm{H}, \mathrm{s}), 0.05(3 \mathrm{H}$, s).

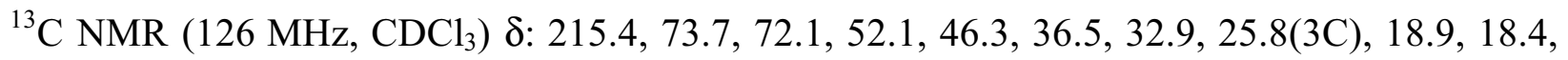
$18.0,17.8,14.1,11.6,-4.39,-4.43$.

Minor isomer:

${ }^{1} \mathrm{H}$ NMR $\left(500 \mathrm{MHz}, \mathrm{CDCl}_{3}\right) \delta: 3.85(1 \mathrm{H}, \mathrm{m}), 3.81(1 \mathrm{H}, \mathrm{m}), 3.12(1 \mathrm{H}, \mathrm{d}, J=2.8 \mathrm{~Hz}), 2.83(1 \mathrm{H}$, $\mathrm{dd}, J=18.0,2.0 \mathrm{~Hz}), 2.67(1 \mathrm{H}, \mathrm{qd}, J=7.0,4.3 \mathrm{~Hz}), 2.46(1 \mathrm{H}, \mathrm{dd}, J=18.0,10.0 \mathrm{~Hz}), 1.67(1 \mathrm{H}$, m), $1.40(2 \mathrm{H}, \mathrm{m}), 1.26(2 \mathrm{H}, \mathrm{m}), 1.05(3 \mathrm{H}, \mathrm{d}, J=7.0 \mathrm{~Hz}), 0.93(3 \mathrm{H}, \mathrm{d}, J=6.8 \mathrm{~Hz}), 0.90(3 \mathrm{H}, \mathrm{d}, J$ $=6.8 \mathrm{~Hz}), 0.88(9 \mathrm{H}, \mathrm{s}), 0.89(3 \mathrm{H}, J=7.0 \mathrm{~Hz}), 0.06(3 \mathrm{H}, \mathrm{s}), 0.05(3 \mathrm{H}, \mathrm{s})$.

${ }^{13} \mathrm{C}$ NMR (126 MHz, $\left.\mathrm{CDCl}_{3}\right) \delta: 215.1,73.8,72.1,52.4,46.3,36.6,32.9,25.8(3 \mathrm{C}), 19.0,18.3$, $18.1,17.8,14.2,11.7,-4.37,-4.42$.

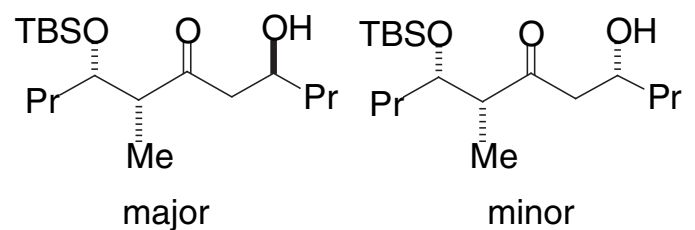

4-(1,1-dimethylethyldimethylsilyloxy)-8-hydroxy-5-methyl-undecan-6-one (11b)

Major isomer: 


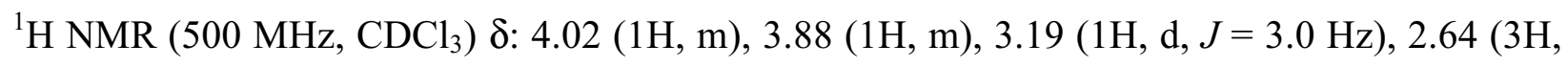
m), $1.38(8 \mathrm{H}, \mathrm{m}), 1.05(3 \mathrm{H}, \mathrm{d}, J=7.0 \mathrm{~Hz}), 0.92(3 \mathrm{H}, \mathrm{t}, J=7.1 \mathrm{~Hz}), 0.89(3 \mathrm{H}, \mathrm{m}), 0.88(9 \mathrm{H}, \mathrm{s})$, $0.06(3 \mathrm{H}, \mathrm{s}), 0.05(3 \mathrm{H}, \mathrm{s})$.

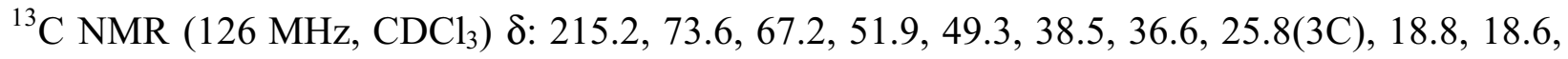
$18.0,14.1,14.0,11.5,-4.36,-4.44$.

Minor isomer:

${ }^{1} \mathrm{H}$ NMR $\left(500 \mathrm{MHz}, \mathrm{CDCl}_{3}\right) \delta: 4.03(1 \mathrm{H}, \mathrm{m}), 3.86(1 \mathrm{H}, \mathrm{m}), 3.21(1 \mathrm{H}, \mathrm{s}), 2.84(1 \mathrm{H}, \mathrm{dd}, J=18.1$, $2.3 \mathrm{~Hz}), 2.66(1 \mathrm{H}, \mathrm{m}), 2.47(1 \mathrm{H}, \mathrm{dd}, J=18.1,9.5 \mathrm{~Hz}), 1.35(8 \mathrm{H}, \mathrm{m}), 1.04(3 \mathrm{H}, \mathrm{d}, J=7.0 \mathrm{~Hz})$, $0.93(3 \mathrm{H}, \mathrm{t}, J=7.1 \mathrm{~Hz}), 0.90(3 \mathrm{H}, \mathrm{t}, J=7.1 \mathrm{~Hz}), 0.89(9 \mathrm{H}, \mathrm{s}), 0.07(6 \mathrm{H}, \mathrm{s})$.

${ }^{13} \mathrm{C}$ NMR (126 MHz, $\left.\mathrm{CDCl}_{3}\right) \delta: 214.9,73.7,67.2,52.2,49.3,38.4,36.6,25.8(3 \mathrm{C}), 18.9,18.6$, $18.0,14.2,14.0,11.6,-4.37,-4.4$.

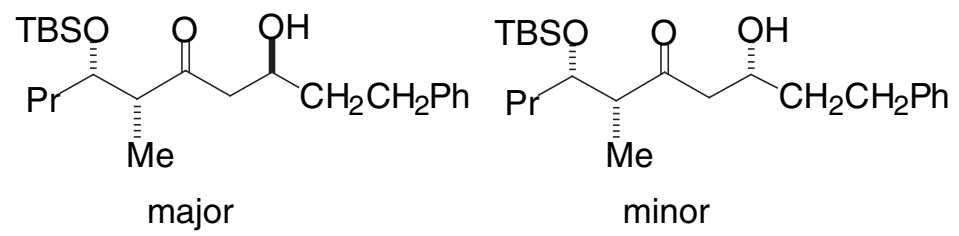

\section{7-(1,1-dimethylethyldimethylsilyloxy)-3-hydroxy-6-methyl-1-phenyl-decan-5-one (11c)}

Major isomer:

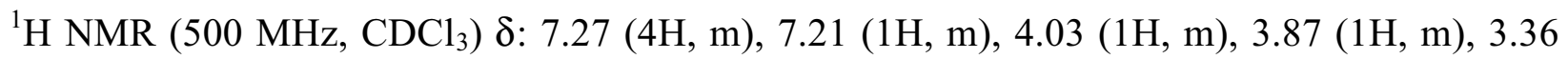
$(1 \mathrm{H}, \mathrm{s}), 2.73(1 \mathrm{H}, \mathrm{dd}, J=18.2,9.2 \mathrm{~Hz}), 2.68(1 \mathrm{H}, \mathrm{m}), 2.63(1 \mathrm{H}, \mathrm{dd}, J=18.2,2.4 \mathrm{~Hz}), 1.84(1 \mathrm{H}$, m), $1.70(1 \mathrm{H}, \mathrm{m}), 1.33(6 \mathrm{H}, \mathrm{m}), 1.05(3 \mathrm{H}, \mathrm{d}, J=7.0 \mathrm{~Hz}), 0.90(3 \mathrm{H}, \mathrm{m}), 0.90(9 \mathrm{H}, \mathrm{s}), 0.08(3 \mathrm{H}, \mathrm{s})$, $0.05(3 \mathrm{H}, \mathrm{s})$.

${ }^{13} \mathrm{C}$ NMR $\left(126 \mathrm{MHz}, \mathrm{CDCl}_{3}\right) \delta: 215.1,141.9,128.6(2 \mathrm{C}), 128.3(2 \mathrm{C}), 125.8,73.6,66.8,51.9$, $49.3,38.0,36.5,31.7,25.8(3 \mathrm{C}), 18.8,18.0,14.1,11.4,-4.4,-4.5$. 
Minor isomer:

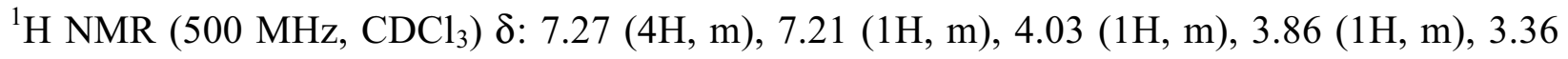
$(1 \mathrm{H}, \mathrm{s}), 2.87(1 \mathrm{H}, \mathrm{dd}, J=18.1,2.3 \mathrm{~Hz}), 2.68(1 \mathrm{H}, \mathrm{m}), 2.52(1 \mathrm{H}, \mathrm{dd}, J=18.2,9.5 \mathrm{~Hz}), 1.84(1 \mathrm{H}$, m), $1.70(1 \mathrm{H}, \mathrm{m}), 1.33(6 \mathrm{H}, \mathrm{m}), 1.04(3 \mathrm{H}, \mathrm{d}, J=7.0 \mathrm{~Hz}), 0.90(3 \mathrm{H}, \mathrm{m}), 0.90(9 \mathrm{H}, \mathrm{s}), 0.08(3 \mathrm{H}, \mathrm{s})$, $0.06(3 \mathrm{H}, \mathrm{s})$.

${ }^{13} \mathrm{C}$ NMR $\left(126 \mathrm{MHz}, \mathrm{CDCl}_{3}\right) \delta: 214.7,141.9,128.6(2 \mathrm{C}), 128.3(2 \mathrm{C}), 125.8,73.7,66.8,52.2$, $49.3,37.9,36.5,31.7,25.8(3 \mathrm{C}), 18.9,18.0,14.0,11.6,-4.4,-4.5$.

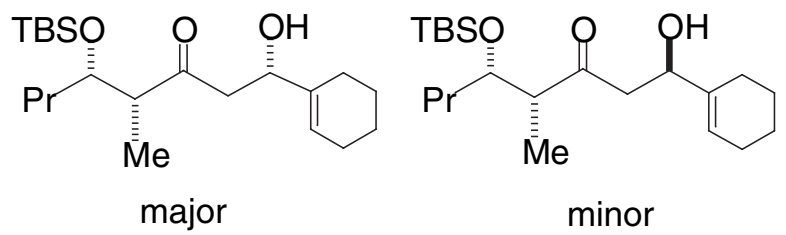

5-(1,1-dimethylethyldimethylsilyloxy)-1-cyclohex-1-enyl-1-hydroxy-4-methyl-octan-3-one (11d)

Major isomer:

${ }^{1} \mathrm{H}$ NMR $\left(500 \mathrm{MHz}, \mathrm{CDCl}_{3}\right) \delta: 5.71(1 \mathrm{H}, \mathrm{s}), 4.41(1 \mathrm{H}, \mathrm{m}), 3.86(1 \mathrm{H}, \mathrm{m}), 3.11(1 \mathrm{H}, \mathrm{s}), 2.86(1 \mathrm{H}$, $\mathrm{dd}, J=17.7,2.6 \mathrm{~Hz}), 2.68(1 \mathrm{H}, \mathrm{m}), 2.65(1 \mathrm{H}, \mathrm{dd}, J=17.7,9.6 \mathrm{~Hz}), 2.02(3 \mathrm{H}, \mathrm{m}), 1.93(1 \mathrm{H}, \mathrm{m})$, $1.59(4 \mathrm{H}, \mathrm{m}), 1.39(4 \mathrm{H}, \mathrm{m}), 1.04(3 \mathrm{H}, \mathrm{d}, J=7.0 \mathrm{~Hz}), 0.90(3 \mathrm{H}, \mathrm{t}, J=7.0 \mathrm{~Hz}), 0.89(9 \mathrm{H}, \mathrm{s}), 0.07$ $(6 \mathrm{H}, \mathrm{s})$.

${ }^{13} \mathrm{C} \mathrm{NMR}\left(126 \mathrm{MHz}, \mathrm{CDCl}_{3}\right) \delta: 214.5,138.2,122.9,73.7,71.6,52.3,48.0,36.6,25.8(3 \mathrm{C}), 24.8$, $24.2,22.54,22.47,18.9,18.0,14.1,11.6,-4.4,-4.4$.

Minor isomer: 
${ }^{1} \mathrm{H}$ NMR $\left(500 \mathrm{MHz}, \mathrm{CDCl}_{3}\right) \delta: 5.71(1 \mathrm{H}, \mathrm{s}), 4.38(1 \mathrm{H}, \mathrm{m}), 3.90(1 \mathrm{H}, \mathrm{m}), 3.11(1 \mathrm{H}, \mathrm{s}), 2.82(1 \mathrm{H}$, dd, $J=17.7,9.5 \mathrm{~Hz}), 2.68(1 \mathrm{H}, \mathrm{m}), 2.66(1 \mathrm{H}, \mathrm{dd}, J=17.7,2.6 \mathrm{~Hz}), 2.02(3 \mathrm{H}, \mathrm{m}), 1.93(1 \mathrm{H}, \mathrm{m})$, $1.59(4 \mathrm{H}, \mathrm{m}), 1.39(4 \mathrm{H}, \mathrm{m}), 1.06(3 \mathrm{H}, \mathrm{d}, J=7.0 \mathrm{~Hz}), 0.89(3 \mathrm{H}, \mathrm{m}), 0.89(9 \mathrm{H}, \mathrm{s}), 0.07(3 \mathrm{H}, \mathrm{s})$, $0.06(3 \mathrm{H}, \mathrm{s})$.

${ }^{13} \mathrm{C} \mathrm{NMR}\left(126 \mathrm{MHz}, \mathrm{CDCl}_{3}\right) \delta: 214.9,138.1,123.0,73.6,71.7,52.0,47.9,36.6,25.8(3 \mathrm{C}), 24.8$, $24.3,22.54,22.47,18.8,18.0,14.1,11.5,-4.4,-4-4$

\section{General procedure for the non-aldol aldol Mukaiyama reaction with $\mathrm{BF}_{3} \cdot \mathbf{O E t}_{2}$}

In an oven dried $10 \mathrm{~mL}$ flask was placed the epoxy alcohol 5' (50 mg), dichloromethane (2 $\mathrm{mL})$ and powdered $4 \AA$ molecular sieves. The solution was cooled to $-40{ }^{\circ} \mathrm{C}$ and $i \operatorname{Pr}_{2} \mathrm{NEt}(1.35$ eq.) was added followed by $t$-butyldimethylsilyl triflate (1.3 eq.). The reaction mixture was stirred at $-40{ }^{\circ} \mathrm{C}$ for $2 \mathrm{~h}$ and the temperature was allowed to rise to $-20{ }^{\circ} \mathrm{C}$. The flask was then left overnight in the freezer at $-16^{\circ} \mathrm{C}$. The reaction was cooled to $-78^{\circ} \mathrm{C}$ and the aldehyde $(1.5$ eq. $)$ and $\mathrm{BF}_{3} \cdot \mathrm{OEt}_{2}\left(1.5\right.$ eq.) were added. The reaction was stirred $30 \mathrm{~min}$ at $-78^{\circ} \mathrm{C}$ and was quenched at that temperature with sat. $\mathrm{NaHCO}_{3}$. It was extracted with dichloromethane and washed with sat. $\mathrm{NaHCO}_{3}$ and brine. The organic phase was dried over $\mathrm{MgSO}_{4}$, filtered and concentrated in vacuo to give a yellowish liquid. The crude ketone was purified by flash column chromatography ( $\mathrm{SiO}_{2}$, pentane/ether: $9 / 1$ to $85 / 15$ to $\left.4 / 1\right)$.
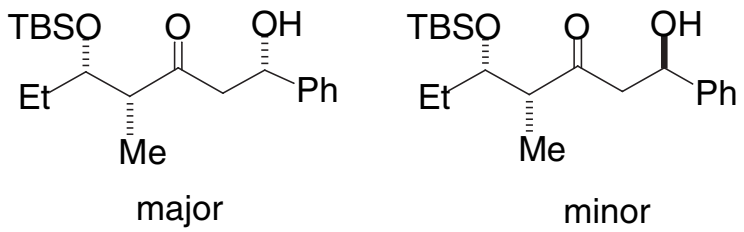

\section{5-(1,1-dimethylethyldimethylsilyloxy)-1-hydroxy-4-methyl-1-phenyl-octan-3-one (12a)}

Major isomer: 


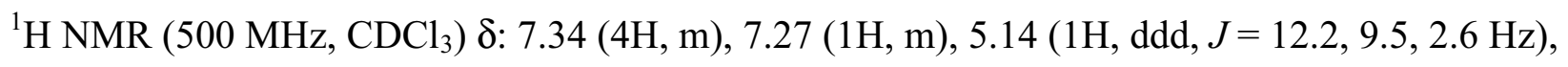
$3.87(1 \mathrm{H}, \mathrm{dd}, J=10.8,5.9 \mathrm{~Hz}), 3.51(1 \mathrm{H}, \mathrm{bs}), 3.00(1 \mathrm{H}, \mathrm{dd}, J=18.0,9.5 \mathrm{~Hz}), 2.84(1 \mathrm{H}, \mathrm{dd}, J=$ 18.0, $2.9 \mathrm{~Hz}), 2.66(1 \mathrm{H}, \mathrm{qd}, J=7.0,4.7 \mathrm{~Hz}), 1.50(1 \mathrm{H}, \mathrm{m}) .1 .37(1 \mathrm{H}, \mathrm{m}), 1.07(3 \mathrm{H}, \mathrm{d}, J=7.0$ Hz), $0.88(9 \mathrm{H}, \mathrm{s}), 0.84(3 \mathrm{H}, \mathrm{t}, J=7.4 \mathrm{~Hz}), 0.07(3 \mathrm{H}, \mathrm{s}), 0.05(3 \mathrm{H}, \mathrm{s})$.

${ }^{13} \mathrm{C}$ NMR (126 MHz, $\left.\mathrm{CDCl}_{3}\right) \delta: 214.3,142.9,128.5(2 \mathrm{C}), 127.5,125.7(2 \mathrm{C}), 74.7,69.9,51.4$, $51.1,27.3,25.8(3 \mathrm{C}), 18.0,11.3,9.8,-4.3,-4.5$.

Minor isomer:

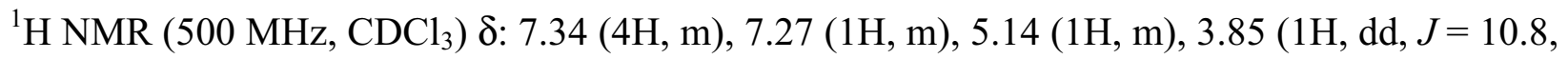
$5.8 \mathrm{~Hz}), 3.47(1 \mathrm{H}, \mathrm{bs}), 3.02(1 \mathrm{H}, \mathrm{dd}, J=17.9,2.7 \mathrm{~Hz}), 2.83(1 \mathrm{H}, \mathrm{dd}, J=18.0,8.9 \mathrm{~Hz}), 2.66(1 \mathrm{H}$, m), $1.50(1 \mathrm{H}, \mathrm{m}) .1 .37(1 \mathrm{H}, \mathrm{m}), 1.06(3 \mathrm{H}, \mathrm{d}, J=6.8 \mathrm{~Hz}), 0.87(9 \mathrm{H}, \mathrm{s}), 0.84(3 \mathrm{H}, \mathrm{t}, J=7.5 \mathrm{~Hz})$, $0.06(3 \mathrm{H}, \mathrm{s}), 0.05(3 \mathrm{H}, \mathrm{s})$.

${ }^{13} \mathrm{C}$ NMR $\left(126 \mathrm{MHz}, \mathrm{CDCl}_{3}\right) \delta: 213.9,142.9,128.5(2 \mathrm{C}), 127.5,125.6(2 \mathrm{C}), 74.8,69.8,51.8$, $51.1,27.3,25.8(3 \mathrm{C}), 18.0,11.4,9.9,-4.3,-4.5$.

For the mixture of diastereomer:

IR (neat): 3470, 2958, 2930, 2857, 705, 1462, 1255, $8361 \mathrm{~cm}^{-1}$. HRMS (EI + voltage, $m / z): 351.234827$, calc for $\mathrm{C}_{20} \mathrm{H}_{35} \mathrm{O}_{3} \mathrm{Si}(\mathrm{M}+\mathrm{H})^{+} 351.235549$. LRMS (EI, $m / z):$ 293, 107.

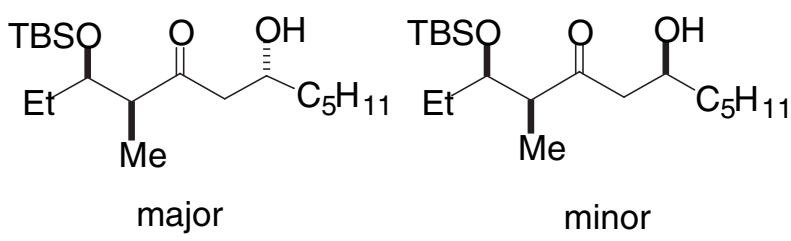

3-(1,1-dimethylethyldimethylsilyloxy)-7-hydroxy-4-methyl-dodecan-5-one (12b) Major isomer: 
${ }^{1} \mathrm{H}$ NMR $\left(500 \mathrm{MHz}, \mathrm{CDCl}_{3}\right) \delta: 4.00(1 \mathrm{H}, \mathrm{m}), 3.83(1 \mathrm{H}, \mathrm{m}), 3.18(1 \mathrm{H}, \mathrm{d}, J=3.1 \mathrm{~Hz}), 2.66(1 \mathrm{H}$, qd, $J=6.9,4.8 \mathrm{~Hz}), 2.64(2 \mathrm{H}, \mathrm{m}), 1.48(2 \mathrm{H}, \mathrm{m}), 1.28(8 \mathrm{H}, \mathrm{m}), 1.06(3 \mathrm{H}, \mathrm{d}, J=7.0 \mathrm{~Hz}), 0.89$ $(9 \mathrm{H}, \mathrm{s}), 0.89(3 \mathrm{H}, \mathrm{m}), 0.86(3 \mathrm{H}, \mathrm{t}, J=7.5 \mathrm{~Hz}), 0.06(3 \mathrm{H}, \mathrm{s}), 0.05(3 \mathrm{H}, \mathrm{s})$.

${ }^{13} \mathrm{C}$ NMR (126 MHz, $\left.\mathrm{CDCl}_{3}\right) \delta: 215.3,74.9,67.5,51.5,49.2,36.3,31.8,27.2,25.8(3 \mathrm{C}), 25.1$, $22.6,18.1,14.0,11.5,9.9,-4.3,-4.5$.

IR (neat): $3473,2931,1704,1463,1256 \mathrm{~cm}^{-1}$.

$[\alpha]_{D}^{20}=+7.0^{\circ}\left(\mathrm{c}=1.0, \mathrm{CHCl}_{3}\right)$

Minor isomer:

${ }^{1} \mathrm{H}$ NMR $\left(500 \mathrm{MHz}, \mathrm{CDCl}_{3}\right) \delta: 4.02(1 \mathrm{H}, \mathrm{m}), 3.81(1 \mathrm{H}, \mathrm{m}), 3.16(1 \mathrm{H}, \mathrm{d}, J=3.1 \mathrm{~Hz}), 2.83(1 \mathrm{H}$, $\mathrm{dd}, J=18.1,2.3 \mathrm{~Hz}), 2.67(1 \mathrm{H}, \mathrm{qd}, J=7.0,4.8 \mathrm{~Hz}), 2.47(1 \mathrm{H}, \mathrm{dd}, J=18.1,9.5 \mathrm{~Hz}), 1.40(10 \mathrm{H}$, m), $1.04(3 \mathrm{H}, \mathrm{d}, J=7.0 \mathrm{~Hz}), 0.89(9 \mathrm{H}, \mathrm{s}), 0.89(3 \mathrm{H}, \mathrm{m}), 0.86(3 \mathrm{H}, \mathrm{m}), 0.06(3 \mathrm{H}, \mathrm{s}), 0.06(3 \mathrm{H}, \mathrm{s})$.

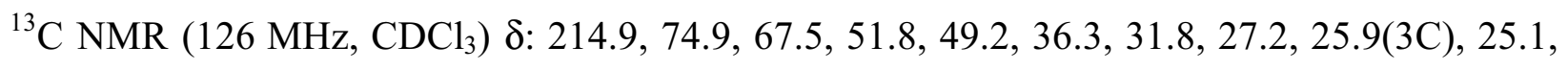
$22.6,18.1,14.0,11.7,10.0,-4.3,-4.5$.

IR (neat): $3458,2930,1705,1463,1256 \mathrm{~cm}^{-1}$.

HRMS (EI, $m / z): 345.282598$, calc for $\mathrm{C}_{19} \mathrm{H}_{40} \mathrm{O}_{3} \mathrm{Si}(\mathrm{M}+\mathrm{H})^{+} 345.282499$.

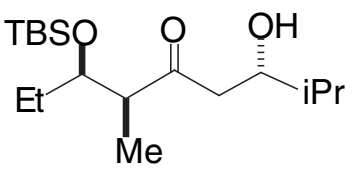

major

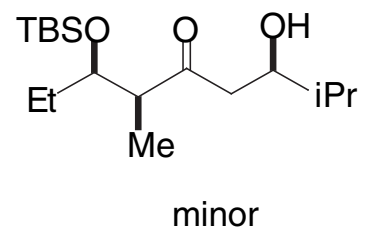

minor

\section{7-(1,1-dimethylethyldimethylsilyloxy)-3-hydroxy-2,6-dimethyl-nonan-5-one (12c)}

Major isomer:

${ }^{1} \mathrm{H}$ NMR (500 MHz, $\left.\mathrm{CDCl}_{3}\right) \delta: 3.83(1 \mathrm{H}, \mathrm{m}), 3.78(1 \mathrm{H}, \mathrm{m}), 3.14(1 \mathrm{H}, \mathrm{d}, J=3.1 \mathrm{~Hz}), 2.68(1 \mathrm{H}$, qd, $J=7.0,4.8 \mathrm{~Hz}), 2.64(1 \mathrm{H}, \mathrm{d}, J=4.7 \mathrm{~Hz}), 2.63(1 \mathrm{H}, \mathrm{d}, J=7.3 \mathrm{~Hz}), 1.67(1 \mathrm{H}, \mathrm{m}), 1.49(1 \mathrm{H}$, 
m), $1.37(1 \mathrm{H}, \mathrm{m}), 1.06(3 \mathrm{H}, \mathrm{d}, J=7.0 \mathrm{~Hz}), 0.93(3 \mathrm{H}, \mathrm{d}, J=6.8 \mathrm{~Hz}), 0.91(3 \mathrm{H}, \mathrm{d}, J=6.8 \mathrm{~Hz})$, $0.89(9 \mathrm{H}, \mathrm{s}), 0.87(3 \mathrm{H}, J=7.5 \mathrm{~Hz}), 0.06(3 \mathrm{H}, \mathrm{s}), 0.05(3 \mathrm{H}, \mathrm{s})$.

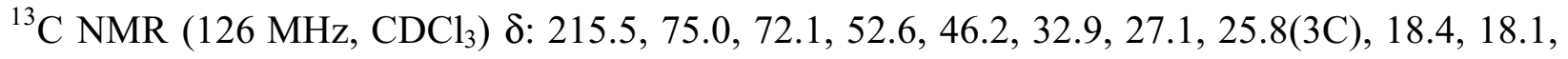
$17.8,11.6,9.9,-4.3,-4.5$.

IR (neat): 3508, 2959, 1703, 1464, $1255 \mathrm{~cm}^{-1}$.

HRMS (EI, $m / z): 259.1733$, calc for $\mathrm{C}_{13} \mathrm{H}_{27} \mathrm{O}_{3} \mathrm{Si}(\mathrm{M}-t \mathrm{Bu})^{+} 259.1729$.

Minor isomer:

${ }^{1} \mathrm{H}$ NMR (500 MHz, $\left.\mathrm{CDCl}_{3}\right) \delta: 3.81(2 \mathrm{H}, \mathrm{m}), 3.10(1 \mathrm{H}, \mathrm{s}), 2.81(1 \mathrm{H}, \mathrm{dd}, J=17.9,2.0 \mathrm{~Hz}), 2.68$ $(1 \mathrm{H}, \mathrm{qd}, J=7.0,4.6 \mathrm{~Hz}), 2.47(1 \mathrm{H}, \mathrm{dd}, J=17.9,9.9 \mathrm{~Hz}), 1.67(1 \mathrm{H}, \mathrm{m}), 1.50(1 \mathrm{H}, \mathrm{m}), 1.31(1 \mathrm{H}$, m), $1.05(3 \mathrm{H}, \mathrm{d}, J=7.0 \mathrm{~Hz}), 0.93(3 \mathrm{H}, \mathrm{d}, J=6.8 \mathrm{~Hz}), 0.91(3 \mathrm{H}, \mathrm{d}, J=6.8 \mathrm{~Hz}), 0.89(9 \mathrm{H}, \mathrm{s}), 0.88$ $(3 \mathrm{H}, \mathrm{m}), 0.06(6 \mathrm{H}, \mathrm{s})$.

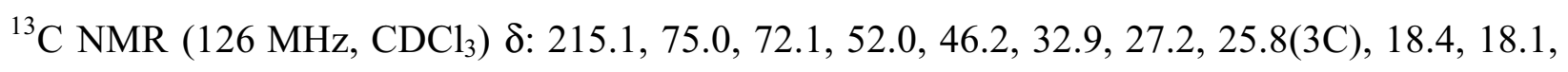
$17.8,11.7,10.0,-4.3,-4.5$.

IR (neat): $3484,2960,1705,1464,1256 \mathrm{~cm}^{-1}$.<smiles>CCC(O[R15](C)(C)C)C(C)C(=O)CC(O)CCc1ccccc1</smiles>

\section{7-(1,1-dimethylethyldimethylsilyloxy)-3-hydroxy-6-methyl-1-phenyl-nonan-5-one (12d)}

NMR of mixture:

Major isomer:

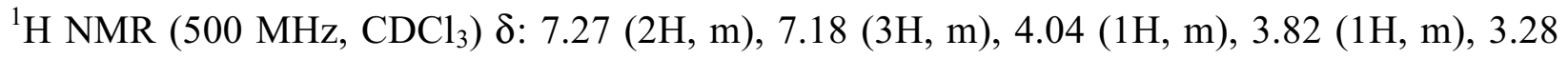
(1H, bs), $2.81(1 \mathrm{H}, \mathrm{m}), 2.70(1 \mathrm{H}, \mathrm{m}), 2.62(1 \mathrm{H}, \mathrm{dd}, J=18.2,2.7 \mathrm{~Hz}), 1.81(1 \mathrm{H}, \mathrm{m}), 1.68(1 \mathrm{H}$, 
m), $1.49(1 \mathrm{H}, \mathrm{m}), 1.36(1 \mathrm{H}, \mathrm{m}), 1.05(3 \mathrm{H}, \mathrm{d}, J=7.0 \mathrm{~Hz}), 0.89(9 \mathrm{H}, \mathrm{s}), 0.88(3 \mathrm{H}, \mathrm{t}, J=7.5 \mathrm{~Hz})$, $0.06(3 \mathrm{H}, \mathrm{s}), 0.04(3 \mathrm{H}, \mathrm{s})$.

${ }^{13} \mathrm{C}$ NMR (126 MHz, $\left.\mathrm{CDCl}_{3}\right) \delta: 215.1,142.0,128.5(2 \mathrm{C}), 128.4(2 \mathrm{C}), 125.8,74.9,66.8,51.5$, $49.2,38.0,31.7,27.1,25.8(3 \mathrm{C}), 18.0,11.5,9.9,-4.3,-4.5$.

Minor isomer:

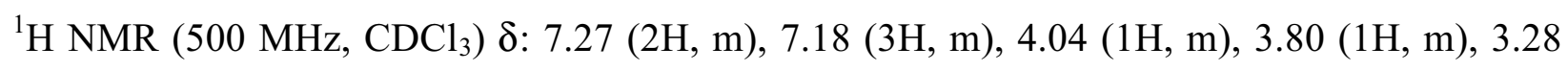
(1H, bs), $2.84(1 \mathrm{H}, \mathrm{dd}, J=18.2,2.3 \mathrm{~Hz}), 2.70(1 \mathrm{H}, \mathrm{m}), 2.55(1 \mathrm{H}, \mathrm{dd}, J=18.2,9.5 \mathrm{~Hz}), 1.81(1 \mathrm{H}$, m), $1.68(1 \mathrm{H}, \mathrm{m}), 1.49(1 \mathrm{H}, \mathrm{m}), 1.36(1 \mathrm{H}, \mathrm{m}), 1.04(3 \mathrm{H}, \mathrm{d}, J=7.0 \mathrm{~Hz}), 0.89(9 \mathrm{H}, \mathrm{s}), 0.88(3 \mathrm{H}$, m), $0.06(3 \mathrm{H}, \mathrm{s}), 0.04(3 \mathrm{H}, \mathrm{s})$.

IR (neat): 3458, 3062, 3027, 2930, 1702, 1455, $1255 \mathrm{~cm}^{-1}$.
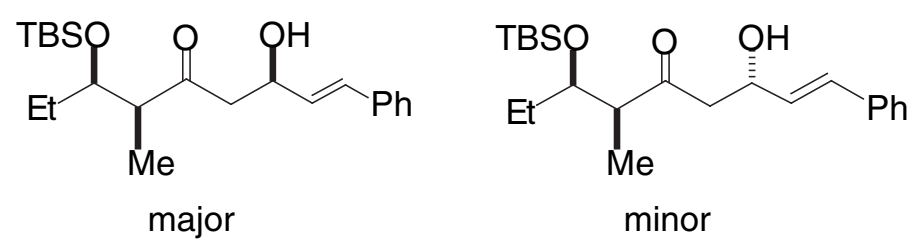

7-(1,1-dimethylethyldimethylsilyloxy)-3-hydroxy-6-methyl-1-phenyl-non-1-en-5-one (12e)

Major isomer:

${ }^{1} \mathrm{H}$ NMR (500 MHz, $\left.\mathrm{CDCl}_{3}\right) \delta: 7.37(2 \mathrm{H}, \mathrm{m}), 7.30(2 \mathrm{H}, \mathrm{m}), 7.25(1 \mathrm{H}, \mathrm{m}), 6.64(1 \mathrm{H}, \mathrm{d}, J=15.9$ Hz), $6.20(1 \mathrm{H}, \mathrm{dd}, J=15.9,6.0 \mathrm{~Hz}), 4.73(1 \mathrm{H}, \mathrm{m}), 3.85(1 \mathrm{H}, \mathrm{m}), 3.33(1 \mathrm{H}, \mathrm{d}, J=3.5 \mathrm{~Hz}), 2.87$ $(1 \mathrm{H}, \mathrm{dd}, J=18.0,8.8 \mathrm{~Hz}), 2.79(1 \mathrm{H}, \mathrm{dd}, J=17.9,3.2 \mathrm{~Hz}), 2.70(1 \mathrm{H}, \mathrm{m}), 1.51(1 \mathrm{H}, \mathrm{m}), 1.38(1 \mathrm{H}$, m), $1.08(3 \mathrm{H}, \mathrm{d}, J=7.0 \mathrm{~Hz}), 0.89(9 \mathrm{H}, \mathrm{s}), 0.87(3 \mathrm{H}, \mathrm{t}, J=7.4 \mathrm{~Hz}), 0.07(6 \mathrm{H}, \mathrm{s})$.

${ }^{13} \mathrm{C} \mathrm{NMR}\left(126 \mathrm{MHz}, \mathrm{CDCl}_{3}\right)$ $\delta: 214.3,136.7,130.25,130.20,128.5(2 \mathrm{C}), 127.6,126.5(2 \mathrm{C}), 74.8$, $68.4,51.5,49.1,27.2,25.9(3 \mathrm{C}), 18.1,11.5,9.8,-4.3,-4.5$. 
Minor isomer:

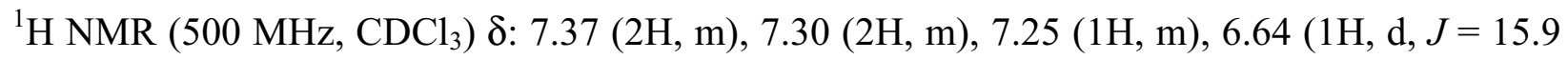
Hz), $6.20(1 \mathrm{H}, \mathrm{dd}, J=15.9,6.0 \mathrm{~Hz}), 4.73(1 \mathrm{H}, \mathrm{m}), 3.85(1 \mathrm{H}, \mathrm{m}), 3.30(1 \mathrm{H}, \mathrm{d}, J=3.4 \mathrm{~Hz}), 2.96$ $(1 \mathrm{H}, \mathrm{dd}, J=17.9,2.9 \mathrm{~Hz}), 2.71(1 \mathrm{H}, \mathrm{dd}, J=17.9,8.9 \mathrm{~Hz}), 2.70(1 \mathrm{H}, \mathrm{m}), 1.51(1 \mathrm{H}, \mathrm{m}), 1.38(1 \mathrm{H}$, m), $1.07(3 \mathrm{H}, \mathrm{d}, J=7.1 \mathrm{~Hz}), 0.89(9 \mathrm{H}, \mathrm{s}), 0.87(3 \mathrm{H}, \mathrm{t}, J=7.4 \mathrm{~Hz}), 0.06(6 \mathrm{H}, \mathrm{s})$.

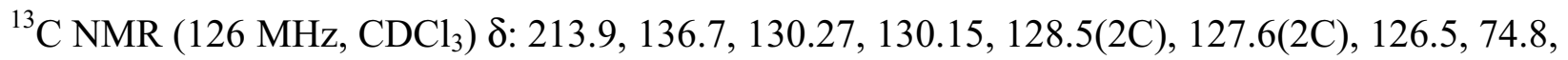
$68.3,51.8,49.1,27.2,25.9(3 \mathrm{C}), 18.1,11.6,10.0,-4.3,-4.4$.

IR (neat): $3427,3027,1705,1255 \mathrm{~cm}^{-1}$.

HRMS (EI, $m / z): 376.24255$, calc for $\mathrm{C}_{22} \mathrm{H}_{36} \mathrm{O}_{3} \mathrm{Si}(\mathrm{M})^{+} 376.24337$.

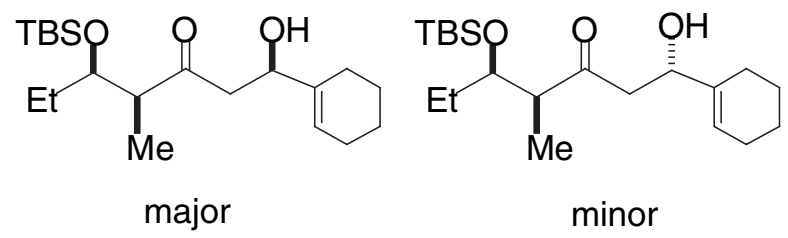

5-(1,1-dimethylethyldimethylsilyloxy)-1-cyclohex-1-enyl-1-hydroxy-4-methyl-heptan-3-one (12f)

NMR of mixture:

Major isomer:

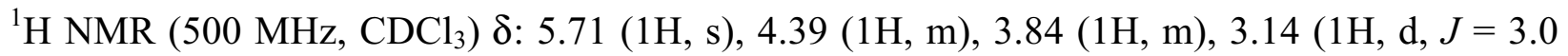
Hz), $2.80(1 \mathrm{H}, \mathrm{dd}, J=17.8,9.6 \mathrm{~Hz}), 2.67(2 \mathrm{H}, \mathrm{m}), 2.06(3 \mathrm{H}, \mathrm{m}), 1.92(1 \mathrm{H}, \mathrm{m}), 1.56(3 \mathrm{H}, \mathrm{m})$, $1.27(3 \mathrm{H}, \mathrm{m}), 1.06(3 \mathrm{H}, \mathrm{d}, J=7.0 \mathrm{~Hz}), 0.89(9 \mathrm{H}, \mathrm{s}), 0.87(3 \mathrm{H}, \mathrm{t}, J=7.3 \mathrm{~Hz}), 0.06(6 \mathrm{H}, \mathrm{s})$.

${ }^{13} \mathrm{C} \mathrm{NMR}\left(126 \mathrm{MHz}, \mathrm{CDCl}_{3}\right) \delta: 214.9,138.3,123.0,74.8,71.7,51.6,47.8,27.2,25.8(3 \mathrm{C}), 24.9$, $24.26,22.6,22.3,18.1,9.9,-4.3,-4.5$.

Minor isomer: 
${ }^{1} \mathrm{H}$ NMR $\left(500 \mathrm{MHz}, \mathrm{CDCl}_{3}\right) \delta: 5.71(1 \mathrm{H}, \mathrm{s}), 4.39(1 \mathrm{H}, \mathrm{m}), 3.81(1 \mathrm{H}, \mathrm{m}), 3.03(1 \mathrm{H}, \mathrm{d}, J=3.0$ Hz), $2.84(1 \mathrm{H}, \mathrm{dd}, J=17.7,2.5 \mathrm{~Hz}), 2.67(2 \mathrm{H}, \mathrm{m}), 2.02(3 \mathrm{H}, \mathrm{m}), 1.92(1 \mathrm{H}, \mathrm{m}), 1.56(3 \mathrm{H}, \mathrm{m})$, $1.27(3 \mathrm{H}, \mathrm{m}), 1.04(3 \mathrm{H}, \mathrm{d}, J=7.0 \mathrm{~Hz}), 0.89(9 \mathrm{H}, \mathrm{s}), 0.87(3 \mathrm{H}, \mathrm{t}, J=7.4 \mathrm{~Hz}), 0.06(6 \mathrm{H}, \mathrm{s})$.

${ }^{13} \mathrm{C}$ NMR $\left(126 \mathrm{MHz}, \mathrm{CDCl}_{3}\right) \delta: 214.5,138.2,122.9,74.9,71.6,51.9,47.9,27.17,25.8,24.9$, $24.31,22.5,18.1,11.6,-4.3,-4.5$.

IR (neat): 3462, 2930, 1705, 1463, $1255 \mathrm{~cm}^{-1}$.

HRMS (EI + volt scan, $m / z): 354.2598$, calc for $\mathrm{C}_{20} \mathrm{H}_{38} \mathrm{O}_{3} \mathrm{Si}(\mathrm{M})^{+} 354.2590$.

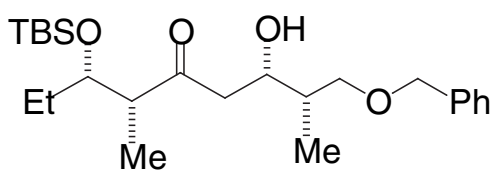

(2S,3R,6R,7S)-1-Benzyloxy-3,7-dihydroxy-2,6-dimethyl-7-(1,1-dimethylethyldimethylsilyloxy)-nonan-5-one (14)

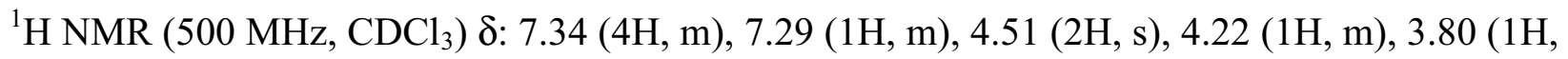
m), $3.51(1 \mathrm{H}, \mathrm{dd}, J=9.1,6.5 \mathrm{~Hz}, 3.47(1 \mathrm{H}, \mathrm{dd}, 9.1,5.2 \mathrm{~Hz}), 3.16(1 \mathrm{H}, \mathrm{d}, J=3.3 \mathrm{~Hz}), 2.71(1 \mathrm{H}$, dd, $J=17.6,3.1 \mathrm{~Hz}), 2.68(1 \mathrm{H}, \mathrm{m}), 2.63(1 \mathrm{H}, \mathrm{d}, J=17.6,9.2 \mathrm{~Hz}), 1.85(1 \mathrm{H}, \mathrm{m}), 1.49(1 \mathrm{H}, \mathrm{m})$, $1.32(1 \mathrm{H}, \mathrm{m}), 1.03(3 \mathrm{H}, \mathrm{t}, J=7.0 \mathrm{~Hz}), 0.95(3 \mathrm{H}, \mathrm{d}, J=7.0 \mathrm{~Hz}), 0.89(9 \mathrm{H}, \mathrm{s}), 0.86(3 \mathrm{H}, \mathrm{t}, J=7.4$ $\mathrm{Hz}), 0.06(3 \mathrm{H}, \mathrm{s}), 0.05(3 \mathrm{H}, \mathrm{s})$.

${ }^{13} \mathrm{C}$ NMR (126 MHz, $\left.\mathrm{CDCl}_{3}\right)$ \&: 214.2, 138.3, 128.4(2C), 127.6(3C), 74.9, 73.6, 73.3, 68.8, 51.8, $46.9,38.0,27.2,25.8(3 \mathrm{C}), 18.1,11.7,11.3,9.9,-4.3,-4.5$.

$[\alpha]_{D}^{20}=-45^{\circ}\left(\mathrm{c}=0.6, \mathrm{CHCl}_{3}\right)$. 


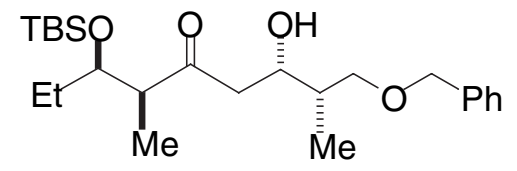

(2S,3S,6S,7R)-1-Benzyloxy-3,7-dihydroxy-2,6-dimethyl-7-(1,1-dimethylethyldimethylsilyloxy)-nonan-5-one (15)

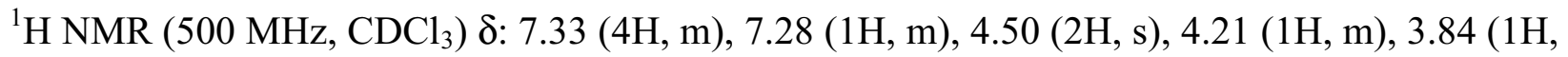
m), $3.51(1 \mathrm{H}, \mathrm{dd}, J=9.1,6.6 \mathrm{~Hz}), 3.45(1 \mathrm{H}, \mathrm{dd}, 9.1,5.2 \mathrm{~Hz}), 3.19(1 \mathrm{H}, \mathrm{d}, J=3.1 \mathrm{~Hz}), 2.74(1 \mathrm{H}$, $\mathrm{dd}, J=17.7,9.7 \mathrm{~Hz}), 2.68(1 \mathrm{H}, \mathrm{qd}, J=7.0,4.9 \mathrm{~Hz}), 2.56(1 \mathrm{H}, \mathrm{d}, J=17.7,2.2 \mathrm{~Hz}), 1.83(1 \mathrm{H}$, m), $1.49(1 \mathrm{H}, \mathrm{m}), 1.37(1 \mathrm{H}, \mathrm{m}), 1.05(3 \mathrm{H}, \mathrm{t}, J=7.0 \mathrm{~Hz}), 0.95(3 \mathrm{H}, \mathrm{d}, J=7.0 \mathrm{~Hz}), 0.88(9 \mathrm{H}, \mathrm{s})$, $0.86(3 \mathrm{H}, \mathrm{t}, J=7.4 \mathrm{~Hz}), 0.06(3 \mathrm{H}, \mathrm{s}), 0.04(3 \mathrm{H}, \mathrm{s})$.

${ }^{13} \mathrm{C}$ NMR (126 MHz, $\left.\mathrm{CDCl}_{3}\right) \delta: 214.6,138.3,128.4(2 \mathrm{C}), 127.6(2 \mathrm{C}), 127.6,74.7,73.4,73.3$, $68.6,51.5,46.9,38.1,27.2,25.9(3 \mathrm{C}), 18.1,11.7,11.3,9.8,-4.3,-4.5$.

IR (neat): 3501, 3065, 3031, 2931, 1704, 1455, 1255.

$[\alpha]_{D}^{20}=+9.1^{\mathrm{o}}\left(\mathrm{c}=0.6, \mathrm{CHCl}_{3}\right)$

\section{Determination of the stereochemistry:}<smiles>CCC(O)C(C)C(O)CC(O)c1ccccc1</smiles>

\section{(1R,3S,4S,5R)-4-Methyl-1-phenyl-heptane-1,3,5-triol (17)}

To a $100 \mathrm{~mL}$ flask containing the ketone 16 (870 mg, $1.87 \mathrm{mmol})$ was added a $2 \mathrm{~mol} \%$ solution of $\mathrm{HF} / \mathrm{CH}_{3} \mathrm{CN}(16 \mathrm{~mL})$. The reaction mixture was stirred at $21^{\circ} \mathrm{C}$ for $90 \mathrm{~min}$. and more $\mathrm{HF} / \mathrm{CH}_{3} \mathrm{CN}(1 \mathrm{~mL})$ was added. The reaction was stirred at $21^{\circ} \mathrm{C}$ for $90 \mathrm{~min}$. The reaction was quenched by adding sat. $\mathrm{NaHCO}_{3}(5 \mathrm{~mL})$. The milky aqueous phase was extracted with ethyl 
acetate $(4 \mathrm{x})$, the combined organic phases were washed with brine $(5 \mathrm{~mL})$ and the organic phase was dried over $\mathrm{MgSO}_{4}$, filtered and concentrated in vacuo to give a yellowish liquid. The crude diol was purified by flash column chromatography $\left(\mathrm{SiO}_{2}\right.$, pentane/ether: $45 / 55$ to $\left.2 / 3\right)$ to give a colorless liquid in $78 \%$ yield as a $4 / 1$ diastereomeric mixture.

To a $100 \mathrm{~mL}$ flask containing the diol $(150 \mathrm{mg}, 0.636 \mathrm{mmol})$ was added tetrahydrofuran $(5.1$ $\mathrm{mL})$ and methanol $(1.3 \mathrm{~mL})$. The solution was cooled to $-78^{\circ} \mathrm{C}$. Triethylborane-THF complex $(1$ $\mathrm{M}$ in tetrahydrofuran, $699 \mu \mathrm{L}, 0.699 \mathrm{mmol}$ ) was added dropwise to the solution and stirred for 35 min followed by sodium borohydride ( $26 \mathrm{mg}, 0.699 \mathrm{mmol}$ ). The reaction was stirred for $90 \mathrm{~min}$ at $-78{ }^{\circ} \mathrm{C}$ and then quenched by adding acetic acid $(0.8 \mathrm{~mL})$ and ethyl acetate $(80 \mathrm{~mL})$. The organic phase was washed with sat. $\mathrm{NaHCO}_{3}(2 \times 1 \mathrm{~mL})$, dried over $\mathrm{MgSO}_{4}$, filtered and concentrated in vacuo to give an oil. The compound was purified by flash column chromatography ( $\mathrm{SiO}_{2}$, pentane/ether: $3 / 7$ to $25 / 75$ to $1 / 4$ ) to give a colorless gue in $50 \%$ yield (76.5 $\mathrm{mg}, 0.32 \mathrm{mmol}$ ) as one major compound.

${ }^{1} \mathrm{H}$ NMR (500 MHz, $\left.\mathrm{CDCl}_{3}\right)$ \&: $7.35(4 \mathrm{H}, \mathrm{m}), 7.28(1 \mathrm{H}, \mathrm{m}), 4.96(1 \mathrm{H}, \mathrm{dd}, J=10.3,2.4 \mathrm{~Hz}), 4.03$ $(1 \mathrm{H}, \mathrm{m}), 3.84(1 \mathrm{H}, \mathrm{m}), 2.02(1 \mathrm{H}, \mathrm{m}), 1.77(1 \mathrm{H}, \mathrm{ddd}, J=14.4,2.1,2.1 \mathrm{~Hz}), 1.53(6 \mathrm{H}, \mathrm{m}), 0.97$ $(3 \mathrm{H}, \mathrm{d}, J=7.1 \mathrm{~Hz}), 0.93(3 \mathrm{H}, \mathrm{t}, J=7.5 \mathrm{~Hz})$.

${ }^{13} \mathrm{C} \mathrm{NMR}\left(126 \mathrm{MHz}, \mathrm{CDCl}_{3}\right)$ \&: 144.4, 128.5(2C), 127.7, 125.6(2C), 77.3, 75.8, 74.2, 44.1, 41.6, $26.8,11.1,10.6$.<smiles>CCC1OC(C)(C)OC(CC(O)c2ccccc2)C1C</smiles><smiles>CCC1C2CCC(CC(O)c3ccccc3)(OC1(C)C)C2(C)C</smiles>

$(1 R, 3 S, 4 S, 5 R)-\beta$-Ethyl-2,2,5-trimethyl-1-phenyl-[1,3]dioxane-4-ethanol (18) 
In a $25 \mathrm{~mL}$ flask the triol $17(60 \mathrm{mg}, 0.25 \mathrm{mmol})$ was dissolved in dichloromethane $(1.5 \mathrm{~mL})$ and 2,2-dimethoxypropane $(62 \mu \mathrm{L}, 0.5 \mathrm{mmol})$ was added to the solution followed by camphorsulfonic acid (cat.). After stirring for $2 \mathrm{~h}$ the starting material remained and more 2,2dimethoxypropane ( $62 \mu \mathrm{L}, 0.5 \mathrm{mmol}$ ) was added followed by camphorsulfonic acid (cat.) This was repeated again after $2 \mathrm{~h}$. The reaction was quenched after 2 hours with a few drops of $\mathrm{NaHCO}_{3}$. The aqueous phase was extracted with ethyl acetate. The combined organic phases were washed with brine, dried over $\mathrm{MgSO}_{4}$, filtrated and concentrated in vacuo to give an oil. Two major diastereomers 18 and 19 were isolated by flash column chromatography for analysis.

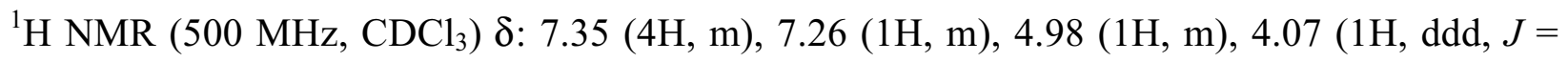
10.5, 2.2, 2.2 Hz), 3.69 (1H, ddd, $J=7.3,7.1,2.2 \mathrm{~Hz}), 3.17(1 \mathrm{H}, \mathrm{d}, J=5.9 \mathrm{~Hz}), 2.16(1 \mathrm{H}, \mathrm{ddd}, J$ $=14.4,10.5,3.4 \mathrm{~Hz}), 1.67(1 \mathrm{H}, \mathrm{ddd}, J=14.4,6.7,2.1 \mathrm{~Hz}), 1.49(1 \mathrm{H}, \mathrm{qdd}, J=13.7,7.4,7.4 \mathrm{~Hz})$, $1.42(3 \mathrm{H}, \mathrm{s}), 1.39(3 \mathrm{H}, \mathrm{s}), 1.34(1 \mathrm{H}, \mathrm{qdd}, J=13.8,7.3,6.4 \mathrm{~Hz}), 1.23(1 \mathrm{H}, \mathrm{qdd}, J=6.9,2.3,2.2$ $\mathrm{Hz}), 0.86(3 \mathrm{H}, \mathrm{d}, J=6.9 \mathrm{~Hz}), 0.84(3 \mathrm{H}, \mathrm{t}, J=7.4 \mathrm{~Hz})$.

${ }^{13} \mathrm{C}$ NMR (126 MHz, $\left.\mathrm{CDCl}_{3}\right)$ \&: 144.7, 128.3(2C), 127.0, 125.6(2C), 99.0, 74.9, 71.5, 70.4, 41.0, $34.8,30.1,25.5,19.8,9.6,4.9$.

IR (neat): 3444, 3062, 3029, $1455,1199 \mathrm{~cm}^{-1}$.

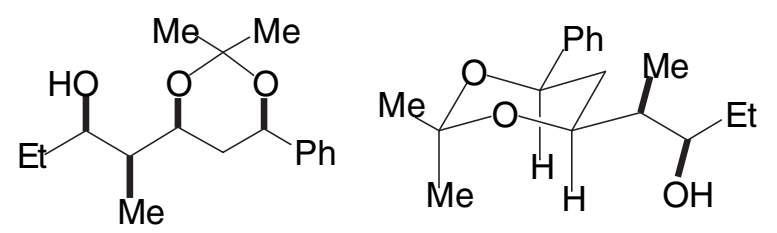

$(\alpha S, \beta R, 4 S, 6 R)-\beta$-Ethyl- $\alpha, 2,2$-trimethyl-6-phenyl-[1,3]dioxane-4-ethanol (19)

${ }^{1} \mathrm{H}$ NMR (500 MHz, $\left.\mathrm{CDCl}_{3}\right)$ \&: $7.37(4 \mathrm{H}, \mathrm{m}), 7.28(1 \mathrm{H}, \mathrm{m}), 4.92(1 \mathrm{H}, \mathrm{dd}, J=11.3,2.9 \mathrm{~Hz}), 4.08$ (1H, ddd, $J=11.4,6.4,2.6 \mathrm{~Hz}), 3.75(1 \mathrm{H}, \mathrm{m}), 2.9(1 \mathrm{H}, \mathrm{m}), 1.69(2 \mathrm{H}, \mathrm{m}), 1.58(1 \mathrm{H}, \mathrm{m}), 1.57$ $(3 \mathrm{H}, \mathrm{s}), 1.51(3 \mathrm{H}, \mathrm{s}), 1.45(2 \mathrm{H}, \mathrm{m}), 0.97(3 \mathrm{H}, \mathrm{t}, J=7.3 \mathrm{~Hz}), 0.91(3 \mathrm{H}, \mathrm{d}, J=7.1 \mathrm{~Hz})$. 
${ }^{13} \mathrm{C}$ NMR (126 MHz, $\left.\mathrm{CDCl}_{3}\right) \delta: 142.2,128.5(2 \mathrm{C}), 127.7,125.9(2 \mathrm{C}), 99.2,74.0,73.3,71.8,41.9$, $37.7,30.3,26.5,19.5,11.00,10.98$.

IR (neat): 3485, 3064, 3030, 1455, $1380 \mathrm{~cm}^{-1}$.

HRMS (EI, $m / z)$ : 279.196583, calc for $\mathrm{C}_{17} \mathrm{H}_{27} \mathrm{O}_{3}(\mathrm{M}+\mathrm{H})^{+} 279.196020$.

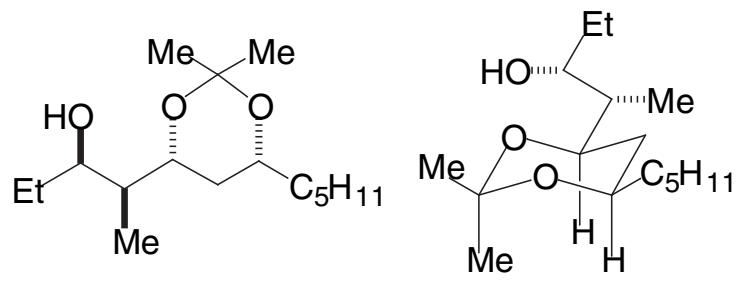

\section{$(\alpha S, \beta R, 4 R, 6 R)-\beta$-ethyl-6-pentyl- $\alpha, 2,2$-trimethyl -[1,3]dioxane-4-ethanol}

${ }^{1} \mathrm{H}$ NMR (500 MHz, $\left.\mathrm{CDCl}_{3}\right) \delta: 3.88(1 \mathrm{H}, \mathrm{ddd}, J=11.6,6.2,2.3 \mathrm{~Hz}), 3.79(1 \mathrm{H}, \mathrm{m}), 3.70(1 \mathrm{H}, \mathrm{m})$, $3.05(1 \mathrm{H}, \mathrm{bs}), 1.62(1 \mathrm{H}, \mathrm{m}), 1.42(3 \mathrm{H}, \mathrm{s}), 1.37(3 \mathrm{H}, \mathrm{s}), 1.35(12 \mathrm{H}, \mathrm{m}), 0.96(3 \mathrm{H}, \mathrm{t}, J=7.3 \mathrm{~Hz})$, $0.88(3 \mathrm{H}, \mathrm{m}), 0.88(3 \mathrm{H}, \mathrm{d}, J=7.0 \mathrm{~Hz})$.

${ }^{13} \mathrm{C}$ NMR (126 MHz, $\left.\mathrm{CDCl}_{3}\right)$ 8: 98.5, 74.1, 73.3, 69.2, 41.9, 36.4, 35.1, 31.8, 30.2, 26.3, 24.6, $22.6,19.5,14.0,11.2,11.0$.

IR (neat): 3521, 2934, 1463, 1380, 1259, $1202 \mathrm{~cm}^{-1}$.

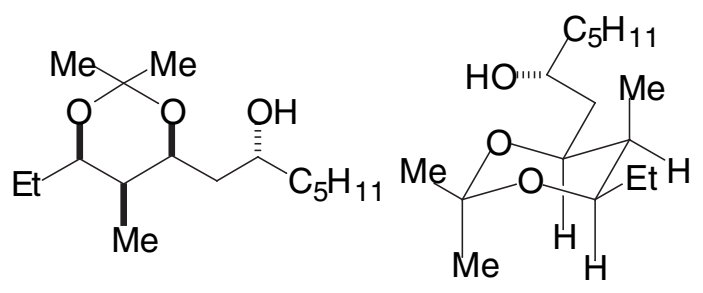

$(\beta R, 4 S, 5 S, 6 \mathrm{R})-6-$-Ethyl-2,2,5-trimethyl- $\beta$-pentyl-[1,3]dioxane-4-ethanol

${ }^{1} \mathrm{H}$ NMR (500 MHz, $\left.\mathrm{CDCl}_{3}\right) \delta: 4.22(1 \mathrm{H}, \mathrm{ddd}, J=10.3,2.2,2.2 \mathrm{~Hz}), 3.79(1 \mathrm{H}, \mathrm{ddd}, J=6.9,6.9$, $2.1 \mathrm{~Hz}), 3.77(1 \mathrm{H}, \mathrm{m}), 2.09(1 \mathrm{H}, \mathrm{s}), 1.81(1 \mathrm{H}, \mathrm{ddd}, J=14.3,10.3,3.0 \mathrm{~Hz}), 1.50(4 \mathrm{H}, \mathrm{m}), 1.45$ 
$(3 \mathrm{H}, \mathrm{s}), 1.39(1 \mathrm{H}, \mathrm{m}), 1.38(3 \mathrm{H}, \mathrm{s}), 1.30(7 \mathrm{H}, \mathrm{m}), 0.89(3 \mathrm{H}, \mathrm{t}, J=7.4 \mathrm{~Hz}), 0.87(3 \mathrm{H}, \mathrm{t}, J=7.4$ $\mathrm{Hz}), 0.84(3 \mathrm{H}, \mathrm{d}, J=6.9 \mathrm{~Hz})$.

${ }^{13} \mathrm{C}$ NMR (126 MHz, $\left.\mathrm{CDCl}_{3}\right) \delta: 98.8,75.0,70.1,69.1,39.6,37.5,35.0,31.9,30.0,25.6,25.5$, 22.6, 19.7, 14.0, 9.6, 4.8 . 